\title{
Evaluación de diferentes niveles de radiación sobre la densidad estomática de tomate (Solanum lycopersicum L.)
}

\section{Evaluation of different radiation levels on tomato stomatal density (Solanum lycopersicum L.)}

\begin{abstract}
William Zárate-Martínez ${ }^{1 *}$ (D)
Marco Antonio Arellano-García ${ }^{2}$,

Francisca Ramírez-Godina $a^{3}$,

Karina Moreno-León ${ }^{4}$ (D)

Dulce Concepción GonzálezSandoval ${ }^{5}$ (D)

${ }^{1}$ Campo Experimental Valles Centrales de Oaxaca, Instituto Nacional de Investigaciones Forestales, Agrícolas y Pecuarias. Melchor Ocampo No. 7, Col. Santo Domingo Barrio Bajo, Etla, CP. 68200 Oaxaca, México.

${ }^{2}$ Departamento de Agroplásticos, Centro de Investigación en Química Aplicada. Blvd. Enrique Reyna No. 140, Col. San José de los Cerritos, CP. 25294, Saltillo, Coahuila. México.

${ }^{3}$ Departamento de Fitomejoramiento, Universidad Autónoma Agraria Antonio Narro. Calz. Antonio Narro No. 1923, Col. Buenavista, CP. 25315, Saltillo, Coahuila. México.

${ }^{4}$ Departamento de Botánica, Universidad Autónoma Agraria Antonio Narro. Calz. Antonio Narro No. 1923, Col. Buenavista, CP. 25315, Saltillo, Coahuila. México.

${ }^{5}$ Departamento de Ciencias del Suelo, Universidad Autónoma Agraria Antonio Narro. Calz. Antonio Narro No. 1923, Col. Buenavista, CP. 25315, Saltillo, Coahuila. México.

*Autor de correspondencia: zarate.william@inifap.gob.mx
\end{abstract}

\section{Nota científica}

Recibido: 14 de abril 2021 Aceptado: 04 de septiembre 2021

Como citar: Zárate-Martínez W, ArellanoGarcía MA, Ramírez-Godina F, MorenoLeón K, González-Sandoval DC (2021) Evaluación de diferentes niveles de radiación sobre la densidad estomática de tomate (Solanum lycopersicum L.). Ecosistemas y Recursos Agropecuarios 8(3): e3009. DOI: 10.19136/era.a8n3.3009
RESUMEN. El objetivo de esta investigación fue evaluar el efecto de distintos niveles de radiación fotosintéticamente activa (RFA) sobre las variables estomáticas en las hojas de plantas de tomate. Los tratamientos fueron los siguientes: $\mathrm{T} 1=25 \%, \mathrm{~T} 2=35 \%, \mathrm{~T} 3=50 \%$, T4 $=60 \%$ y $\mathrm{T} 5=75 \%$ de bloqueo de RFA. Se calculó en la superficie adaxial y abaxial: densidad de estomas, índice estomático, largo y ancho de estomas en las hojas 4 y 9. Se encontró mayor densidad de estomas en la superficie abaxial que en la superficie adaxial, y en la hoja 4 mayor densidad estomática que en la hoja 9; en la hoja 4 el rango de la densidad de estomas fue de 105 a 219 estomas. $\mathrm{mm}^{-2}$ y de 86.9 a 132.9 estomas $\cdot \mathrm{mm}^{-2}$ en las superficies abaxial y adaxial respectivamente, sin embargo, los estomas de mayor tamaño se encontraron en las hojas número 9. La radiación modifica a nivel estomático a las plantas de tomate.

Palabras clave: Abaxial, adaxial, estomas, índice estomático, tamaño de estomas.

ABSTRACT. The objective of this research was to evaluate the effect of different levels of photosynthetically active radiation (RFA) on the stomatal variables in the leaves of tomato plants. The treatments were as follows: $\mathrm{T} 1=25 \%, \mathrm{~T} 2=35 \%, \mathrm{~T} 3=50 \%, \mathrm{~T} 4=60 \%$ and $\mathrm{T} 5=75 \%$ RFA blockade. Stomata density, stomatal index, stomatal length and width were calculated on the adaxial and abaxial surfaces in leaves 4 and 9 . A higher stomatal density was found in the abaxial surface than in the adaxial surface, and in leaf 4 greater stomatal density than in leaf 9 ; in leaf 4 the stomata density range was from 105 to 219 stomata $\cdot \mathrm{mm}^{-2}$ and from 86.9 to 132.9 stomata . $\mathrm{mm}^{-2}$ on the abaxial and adaxial surfaces respectively, however, the largest stomata were found in leaves number 9 . Radiation modifies tomato plants at the stomatal level.

Key words: Abaxial, adaxial, stomata, stomatal index, stomatal size. 


\section{INTRODUCCIÓN}

El tomate (Solanum lycopersicum L.) es una planta originaria de los andes de América del Sur, sin embargo, México es considerado como centro de domesticación de esta especie (Gonzales 2013). Es un cultivo que se encuentra en todo el mundo y representa $72 \%$ del valor de la producción mundial de hortalizas frescas (Hanssen et al. 2010). La superficie destinada a su producción a nivel mundial pasó de 3.9 a 4.8 millones de hectáreas entre 2008 - 2018 y la producción de 140 a 182.3 millones de toneladas en el mismo periodo (FIRA 2019). México ocupa el noveno lugar como productor de esta hortaliza (SIAP 2017). En el 2019 se destinaron 47151 ha para la producción de esta hortaliza, con una producción total de 3.22 millones de toneladas, siendo los principales estados productores Sinaloa, Michoacán, Zacatecas, San Luis Potosí y Baja California Sur con una superficie de 12 256.42, 5 964.83, 2 856.97, 2 840.53 y 2611.65 , respectivamente (SIAP 2020). EI valor promedio anual de las exportaciones durante del 2015 al 2019 fue de 1853 millones de dólares, lo cual, representó 1.57 millones de toneladas (SIAVI 2020).

Las plantas de tomate prefieren suelos ricos en materia orgánica (4-6\%), con buen drenaje, $\mathrm{pH}$ entre 6 y 6.5 para una adecuada disponibilidad de nutrientes, la conductividad eléctrica (C.E.) máxima debe encontrase entre 2.5 y 2.9 , este valor es de gran importancia ya que la salinidad, es una de las principales causas que limitan la productividad de los cultivos (Esteban et al. 2017, Moya et al. 2017). Las temperaturas óptimas para su crecimiento y desarrollo oscilan entre 23 y $26^{\circ} \mathrm{C}$ durante el día y de 13 a $18^{\circ} \mathrm{C}$ durante la noche. Temperaturas entre 10 y $12^{\circ} \mathrm{C}$ detienen el crecimiento de la planta e inferiores a $-2^{\circ} \mathrm{C}$ causan la muerte, debido a que el agua en las células se congela y alcanza una alta densidad, lo que causa ruptura de las células vegetales (INIA 2017).

La radiación luminosa ocupa una pequeña franja del espectro, que va desde los 400 a los 700 $\mathrm{nm}$, se sitúa entre las radiaciones ultravioletas y las infrarrojas, y constituye la radiación fotosintética- mente activa (RFA), medida en $\mu \mathrm{mol} \cdot \mathrm{m}^{-2} \cdot \mathrm{s}^{-1}$ es una fracción de la radiación solar aprovechable por las plantas (Oyarzún et al. 2011, De-las-Rivas 2013). El punto de saturación de luz para las plantas de tomate ocurre entre 1200 y $1400 \mu \mathrm{mol} \cdot \mathrm{m}^{-2} \cdot \mathrm{s}^{-1}$ (Ayari et al. 2000). Por lo que la luz recibida por las plantas debe ser igual o menor al punto de saturación, ya que un incremento de la radiación no significa un incremento en la fotosíntesis (Won-Sub y Sung-Gaun 2012). EI punto de saturación se alcanza cuando, después de cierto nivel de luz la planta ya no puede absorber más dióxido de carbono, lo anterior se relaciona con el tipo de planta que es, las plantas de tomate son C3 (Caballero y de los Santos 2018).

De todos los órganos de las plantas, las hojas son las más sensibles a las condiciones del medio ambiente, por lo que en este órgano se reflejan muchas alteraciones morfológicas y anatómicas (Trewavas 2003, Sánchez-Díaz y Aguirreola 2008). Diversos factores ambientales como bajas y altas temperatura, salinidad, estrés hídrico y radiación, estimulan cambios sobre la densidad estomática (Petrova 2012, Wang et al. 2018). Los estomas son estructuras que forman parte del tejido epidérmico e interrumpen la continuidad de este; un estoma está formado por células epidérmicas especializadas que lo rodean y regulan el tamaño de su abertura (células oclusivas) (Cañizares et al. 2003). Los estomas cumplen un rol de suma importancia en la superficie de las hojas, ya que regulan la perdida de agua y captación del dióxido de carbono durante la fotosíntesis (Apaza et al. 2019). Por lo que una baja densidad estomática induce reducción en la fotosíntesis (Misson et al. 2010).

La principal función de los estomas es la asimilación de $\mathrm{CO}_{2}$ y la pérdida de agua por medio de la transpiración, lo cual, evidencia la importancia de los estomas (Camposeco-Montejo et al 2018). Debido a que los factores ambientales influyen en la tensión del agua en el suelo, planta y atmosfera, estos también influyen en la transpiración, apertura y cierre de estomas (Salisbury y Ross 2000). Entre los factores ambientales más importantes que afectan la transpiración están el déficit de presión de vapor del aire (DPV), la temperatura, la velocidad del viento, la 
concentración de $\mathrm{CO}_{2}$, la disponibilidad de humedad en el suelo y la radiación solar (Sánchez-Díaz y Aguirreolea 2008). La densidad y el tamaño de los estomas interviene en la transpiración, la respiración y la fotosíntesis, por lo tanto, en la acumulación de biomasa y el rendimiento (Ayala et al. 2010). Por lo anterior el objetivo de esta investigación fue evaluar el efecto de diferentes niveles de radiación sobre la densidad de estomas en las hojas de plantas de tomate.

\section{MATERIALES Y MÉTODOS}

\section{Sitio de estudio}

La investigación se realizó en dos etapas: la primera en el campo experimental del Centro de Investigación en Química Aplicada (CIQA). Que se encuentra ubicado en las coordenadas geográficas $25^{\circ} 27^{\prime} 32$ " de latitud Norte y $100^{\circ} 58^{\prime}$ 04" de Iongitud Oeste a una altitud de $1600 \mathrm{msnm}$, con temperatura media anual de $18.2^{\circ} \mathrm{C}$ y precipitación promedio anual de $362.4 \mathrm{~mm}$ (SMN 2018). La segunda etapa se realizó en el laboratorio del Departamento de Fitomejoramiento de la Universidad Autónoma Agraria Antonio Narro (UAAAN), ambas instituciones ubicadas en Saltillo, Coahuila, México.

Como material biológico se utilizó tomate tipo bola del hibrido Yigido. La siembra se realizó en charolas de germinación de poliestireno de 200 cavidades, con sustrato Peat Moss para germinación. Se trasplantó en camas de siembra de $1.2 \mathrm{~m}$ de ancho y $0.30 \mathrm{~m}$ de alto, dentro de túneles de $5 \mathrm{~m}$ de largo, $2.3 \mathrm{~m}$ de ancho y $2.1 \mathrm{~m}$ de alto, 30 días después de la siembra. El cultivo se manejó a un tallo, se podaron yemas laterales y la nutrición fue por medio de un sistema de riego dirigido con la solución de Steiner (Steiner 1961).

Los tratamientos evaluados fueron: T1 $=25 \%$, $\mathrm{T} 2=35 \%$, T3 $=50 \%$, T4 $=60 \%$ y T5 $=75 \%$ de bloqueo de RFA, lo cual, se consiguió debido a las características de las películas plásticas utilizadas. Se trabajó con 20 túneles, divididos en cinco tratamientos y cuatro repeticiones, en cada repetición 20 plantas en un diseño experimental completamente al azar. Para el T1 se utilizó la película tipo SUNTHERM 4
AV (EVA)/C743, para el T2 fue tipo SUNTHERM 4 (EVA)/C702 ambas películas de la casa comercial GINEGAR, para el T3 se utilizaron las dos películas anteriores, las cuales, se encimaron con grapas, para los tratamientos T4 y T5 se utilizaron películas elaboradas en el CIQA, a las que en el proceso de coextrusión se les agregó óxido de titanio a una concentración de 0.5 y $1 \%$ y se les nombró prototipo 06 (PROT-06) y prototipo 05 (PROT-05), respectivamente.

\section{Variables evaluadas}

Para cuantificar la RFA, se instaló en cada repetición un sensor quantum PAR de la marca LICOR, para determinar temperatura y humedad relativa se utilizaron sensores HOBO pro v2, el cual promediaba valores cada cinco minutos, el DPV se determinó con valores de temperatura y humedad relativa con la ecuación propuesta por Rosenberg et al. (1983).

$$
D P V(K P a)=0.61078 \exp \left(\frac{17.269 * T a}{T a+237.3}\right) *\left(1-\frac{H R}{100}\right)
$$

Donde: $\mathrm{DPV}=$ déficit de presión de vapor; $\mathrm{Ta}=$ temperatura del aire $\left({ }^{\circ} \mathrm{C}\right)$ y $\mathrm{HR}=$ humedad relativa (\%). A los 142 días después del trasplante, el cultivo se encontraba en producción y se muestrearon tres plantas al azar por repetición, no se muestrearon plantas de la orilla. En cada planta se seleccionaron dos hojas, la hoja cuatro y la hoja nueve, se consideró como hoja uno, la primer hoja superior totalmente extendida, las hojas seleccionadas tenían la misma orientación (Norte) y altura $(85$ y $160 \mathrm{~cm}$ ).

En laboratorio se tomaron impresiones epidérmicas de la parte media de las hojas seleccionadas, tanto de la superficie adaxial como de la superficie abaxial utilizando poliestireno-xilol líquido, el cual, se aplicó sobre la superficie foliar con un pincel, una vez seco se removió con cinta adhesiva transparente y se pegó en un portaobjetos. De cada impresión se observaron con un microscopio compuesto tres campos al azar a 100X. Por tratamiento, se analizaron 36 campos de la superficie adaxial y 36 campos de la superficie abaxial. De cada observación se determinó densidad estomática (DE) (estomasmm ${ }^{-2}$ ) con 
la siguiente ecuación:

\section{$D E=$ Número de estomas /área del campo visual}

Con campo visual de $0.02544696 \mathrm{~mm}^{-2}$.

El índice estomático (IE), se determinó con la ecuación propuesta por Wilkinson (1979).

$I E=\left(\frac{N^{\circ} \text { de estomas }}{\text { Células epidérmicas }+N^{\circ} \text { de estomas }}\right) \times 100$

La medición de ancho y largo de estomas $(\mu \mathrm{m})$, se realizó de fotografías con el software AxionVision Rel. 4.8

\section{Análisis estadístico}

Los datos obtenidos se concentraron en una hoja de cálculo Excel. Se realizó un análisis de correlación, análisis de varianza y comparación de medias por el método Duncan $(P \leq 0.05)$. El análisis de datos se realizó en el programa estadístico InfoStat (Di Rienzo et al. 2008).

\section{RESULTADOS Y DISCUSIÓN}

El rango de temperatura promedio en los tratamientos fue de 10 a $27{ }^{\circ} \mathrm{C}$, presentando el tratamiento T3 la mayor temperatura. Mientras que la humedad relativa tuvo valores entre 57 a $87 \%$, presentando los tratamientos $\mathrm{T} 1$ y $\mathrm{T} 5$ los valores más bajos. Para DPV el tratamiento T3 presentó los mayores valores antes de las 14 horas y el tratamiento T1 los menores valores después de las 15 horas. Las temperaturas registradas en el T3, fueron mayores a las temperaturas registradas en el $\mathrm{T} 1$, las cuales, se deben a la doble película plástica utilizada en el tratamiento. Los bajos valores de humedad relativa (55 a $57 \%$ ) se registraron entre las 14 y 16 horas, dichos valores se encuentran dentro del rango aceptable para el cultivo de tomate (Sato y Peet 2005). Los tratamientos T1 y T5 recibieron la mayor $\left(900 \mu \mathrm{mol} \cdot \mathrm{m}^{-2} \cdot \mathrm{s}^{-1}\right)$ y menor $\left(300 \mu \mathrm{mol} \cdot \mathrm{m}^{-2} \cdot \mathrm{s}^{-1}\right)$ RFA, respectivamente (Figura 1). Al respecto se sabe que la saturación por RFA se alcanza después de 1 $200 \mu \mathrm{mol} \cdot \mathrm{m}^{-2} \cdot \mathrm{s}^{-1}$ (Ayari et al. 2000). Por lo que los valores máximos de radiación de $900 \mu \mathrm{mol} \cdot \mathrm{m}^{-2} \cdot \mathrm{s}^{-1}$ encontrados en los tratamientos indica que en ningún tratamiento se estresaron las plantas por saturación de RFA.

La superficie abaxial presentó la mayor densidad estomática con respecto a la superficie adaxial, en ambas hojas, mientras que la hoja número 4 presentaron mayor densidad estomática en ambas superficies. Para la densidad de estomas se encontraron diferencias estadísticas significativas entre las hojas evaluadas, la hoja 4 el T5 (219.8 estomas $\cdot \mathrm{mm}^{-2}$ ) y el T1 (105 estomas $\cdot \mathrm{mm}^{-2}$ ) presentaron la mayor y menor densidad estomática. Para la superficie adaxial los tratamientos T1 y T5 presentaron la menor densidad con 86.9 y 88.6 estomas $\cdot \mathrm{mm}^{-2}$, respectivamente. Mientras que para la superficie superficie abaxial en la hoja 9, el tratamiento T1 (95.1 estomas. $\mathrm{mm}^{-2}$ ) presentó la menor densidad estomática; mientras que la menor densidad estomática adaxial los tratamientos T1 y T5 presentaron la menor densidad con 55.8 y 39.4 estomas $\cdot \mathrm{mm}^{-2}$, respectivamente. Al respecto se sabe que altos niveles de radiación reducen la densidad estomática, como se observa en el tratamiento T1 (superior a $800 \mu \mathrm{mol} \cdot \mathrm{m}^{-2} \cdot \mathrm{s}^{-1}$ ) al presentar la menor densidad estomática en superficie abaxial y adaxial en las hojas 4 y 9 (Tabla 1). Sobre la presencia de estomas en la parte abaxial y adaxial del tomate se que es una planta anfiestomáticas (FloresVindas 1999).

La variable densidad celular, presentó diferencias estadísticas significativas entre tratamientos $(p$ $<0.05$ ). En la hoja 4, superficie abaxial, los T1 (538.1 células $\left.\cdot \mathrm{mm}^{-2}\right)$ y T2 (513.5 células $\cdot \mathrm{mm}^{-2}$ ) presentaron la menor densidad celular; en la hoja 9 , la superficie adaxial el T1 (378.9 células $\mathrm{mm}^{-2}$ ) y el T3 (492.1 célulasmm ${ }^{-2}$ ) presentaron la menor y mayor densidad celular respectivamente. Altos niveles de radiación reducen la densidad celular, tal como se observa en los resultados del T1 (superior a 800 $\mu \mathrm{mol} \cdot \mathrm{m}^{-2} \cdot \mathrm{s}^{-1}$ ), fue el tratamiento que recibió mayor radiación y presentó la menor densidad celular. El índice estomático, presentó diferencias estadísticas significativas entre tratamientos $(p<0.05)$. En la hoja 4 , el T5 presentó el mayor índice estomático en la su- 

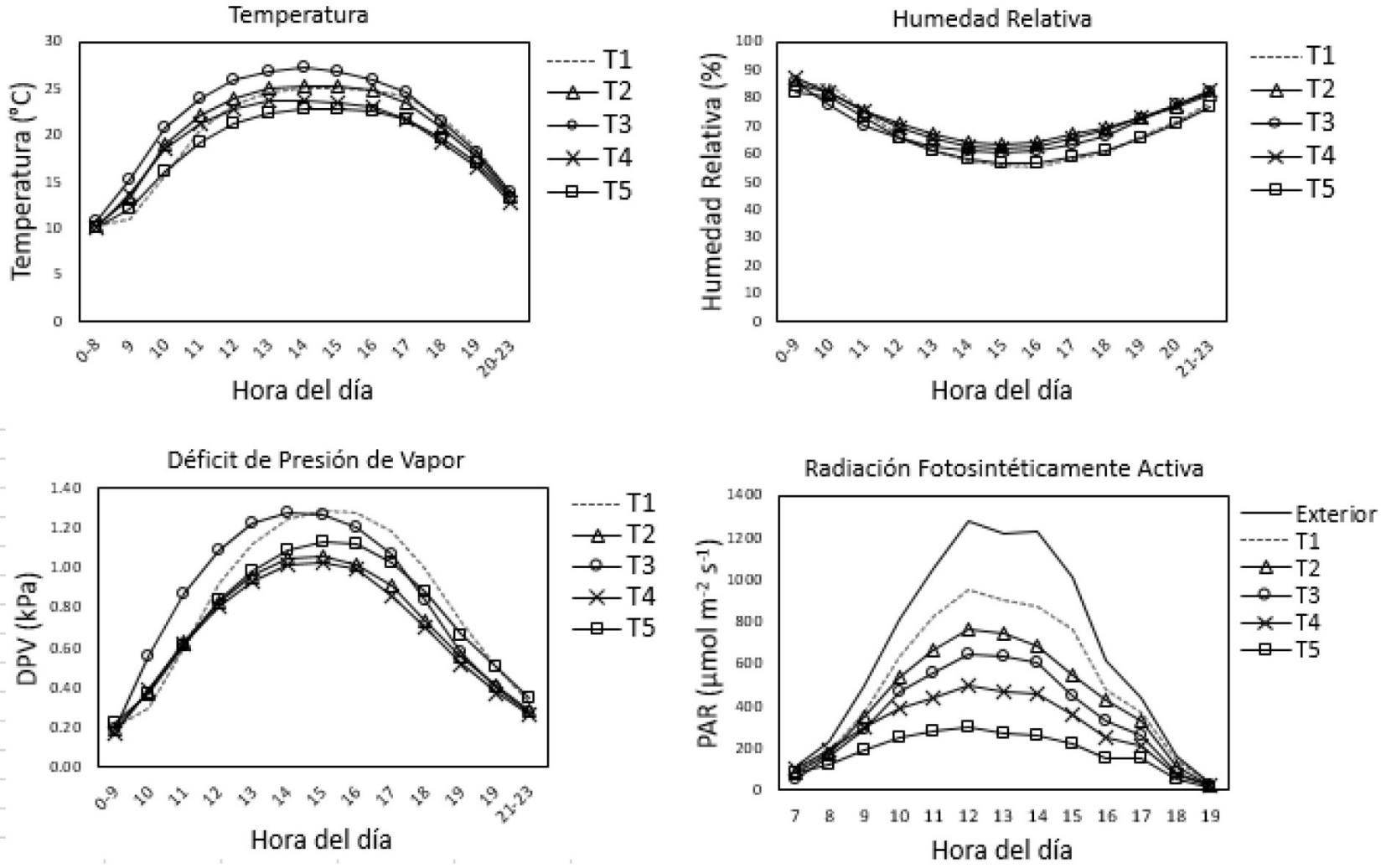

Figura 1. Temperatura, humedad relativa, déficit de presión de vapor y radiación fotosintéticamente activa de cada tratamiento evaluado. T1 $=25 \%$, $\mathrm{T} 2=35 \%, \mathrm{~T} 3=50 \%, \mathrm{~T} 4=60 \%$ y T5 $=75 \%$ del bloqueo de la radiación fotosintéticamente activa (RFA).

Tabla 1. Variables estomáticas en hojas de Solanum lycopersicum.

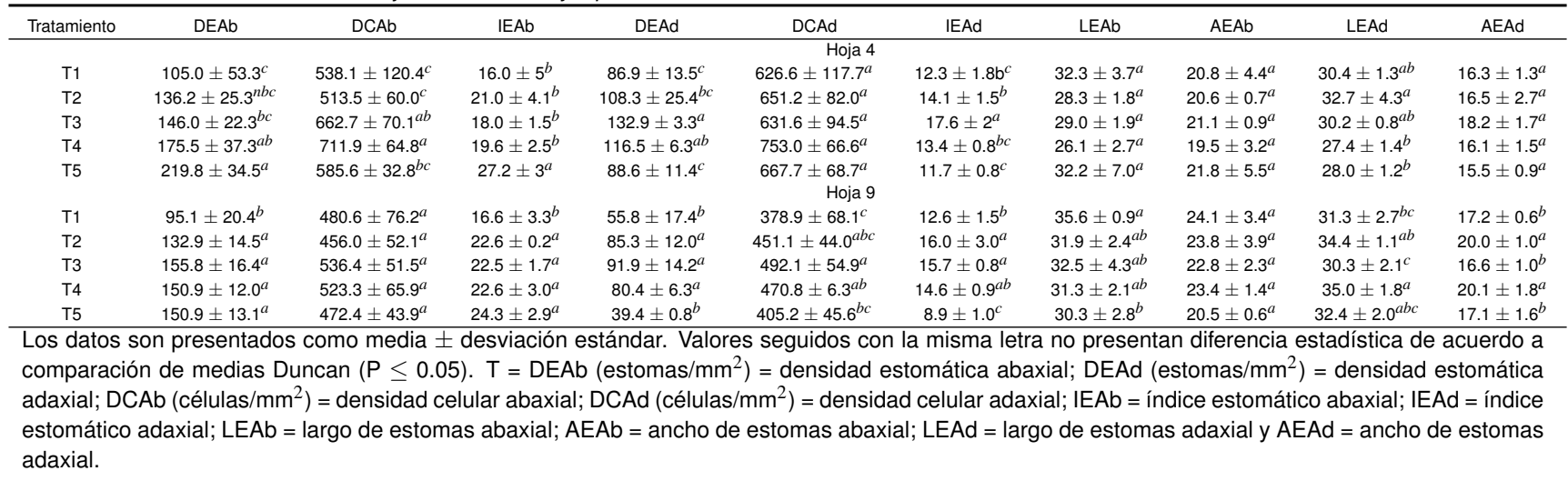

perficie abaxial y el menor índice estomático en la superficie adaxial. En la hoja 9, los T1 y T5 presentaron el menor índice estomático en la superficie abaxial y adaxial respectivamente. En la superficie abaxial de ambas hojas se observó una relación directa entre radiación e índice estomático, en los tratamientos $\mathrm{T} 1$ y T5. Al respecto se sabe que la densidad e índice estomático están influenciados por condiciones ambientales como humedad relativa, salinidad del suelo y radiación solar (Bazaldúa-Muñoz et al. 2008). Sobre lo mismo Brownlee (2001) reporta que altos niveles de radiación originan mayor densidad de estomas, 
sin embargo, en este estudio no se observaron los mismos resultados. Mientras que William y Stimart (2004) afirmaron que la densidad estomática es una característica compleja, sensible al medio ambiente, lo cual, concuerda con los resultados obtenidos en este trabajo, ya que diferentes niveles de radiación, modifican la temperatura y humedad relativa en el medio ambiente y por lo tanto cambios estomáticos en el cultivo. Por lo que someter las plantas de tomate bajo diferentes niveles de radiación estimula modificaciones en la densidad e el índice estomático.

El largo de estomas presentó diferencias estadísticas significativas entre tratamientos $(p<0.05)$. En la hoja 4, el T2 presentó estomas de mayor longitud $(32.7 \mu \mathrm{m})$ en la superficie adaxial. En la hoja 9, superficie abaxial los T1 y T5 presentaron el mayor $(35.6 \mu \mathrm{m})$ y menor $(30.3 \mu \mathrm{m})$ largo de estomas; en la superficie adaxial, los T3 y T4 presentaron el menor $(30.3 \mu \mathrm{m})$ y mayor $(35.0 \mu \mathrm{m})$ largo de estomas respectivamente. La anchura de estomas, solo mostró diferencias estadísticas en la superficie adaxial en las hojas 9, donde, los T2 $(20.0 \mu \mathrm{m})$ y T4 $(20.1 \mu \mathrm{m})$ presentaron los mayores valores.

Según la matriz de correlación, los coeficientes de determinación en la hoja 4 mostraron que hay una excelente correlación entre las variables (IEAb-DEAb) $\left(r^{2}=0.88\right)$ y $(A E A b-L E A b)\left(r^{2}=0.88\right)$. Así también se encontró una buena relación entre las variables (PARDEAb) $\left(r^{2}=0.78\right)$, (PAR-IEAb) $\left(r^{2}=0.64\right)$ y (PARTem) $\left(r^{2}=0.69\right)$. En la hoja 9, las variables que muestran una excelente correlación son las siguientes: (IEAb-DEAb) $\left(r^{2}=0.84\right)$ y (IEAd-DEAd) $(r=0.092)$; las variables (DCAd-DCAb) $(r=0.71)$, (PAR-DEAb) $(r=-0.67)$, (PAR-IEAb) $(r=-0.68)$ y (PAR-Temp) $(r$ $=0.69$ ) presentaron una buena correlación (Tabla 2). Hay una relación directa entre los niveles de radiación y temperatura, sin embargo, la radiación muestra una relación inversa con las variables densidad e índice estomático, lo cual indica, que a mayores niveles de radiación las plantas reducen el número de estomas en sus hojas. La relación entre densidad e índice estomático se observó en la hoja 4 y 9 . Entre la superficie abaxial y adaxial hay estomas de diferentes tamaños así también diferentes densidades, lo anterior se observa en la Figura 2.

La hoja 4 presentó estomas de menor tamaño, debido a que son hojas más jóvenes que las hojas 9, lo cual indica que los estomas serán de mayor tamaño en hojas más desarrolladas. La mayor diferencia estadística en tamaño de estomas se encontró en la superficie adaxial de la hoja 9, algo similar fue reportado por Sánchez (2005) quien afirmó haber encontrado diferencias en el largo de los estomas en la superficie adaxial. El tamaño de estomas es afectado por la radiación.

El nivel de radiación sobre un cultivo de tomate juega un papel importante en sus características estomáticas, lo cual, tiene un efecto sobre las funciones fisiológicas como respiración y fotosíntesis, factores que influyen en el rendimiento de un cultivo. Diferentes niveles de radiación modifican la densidad e índice estomático en las hojas de tomate. Las hojas más jóvenes presentan mayor densidad de estomas, sin embargo, estas hojas presentan estomas de menor tamaño. Altos niveles de radiación originan menor densidad e índice estomático en las hojas de tomate; así también alta radiación provocó estomas de mayor tamaño. La radiación es un factor que modifica a nivel estomático a las plantas de tomate.

\section{AGRADECIMIENTOS}

Al Consejo Nacional de Ciencia y Tecnología (CONACYT), al Centro de Investigación en Química Aplicada (CIQA) y la Universidad Autónoma Agraria Antonio Narro (UAAAN). 


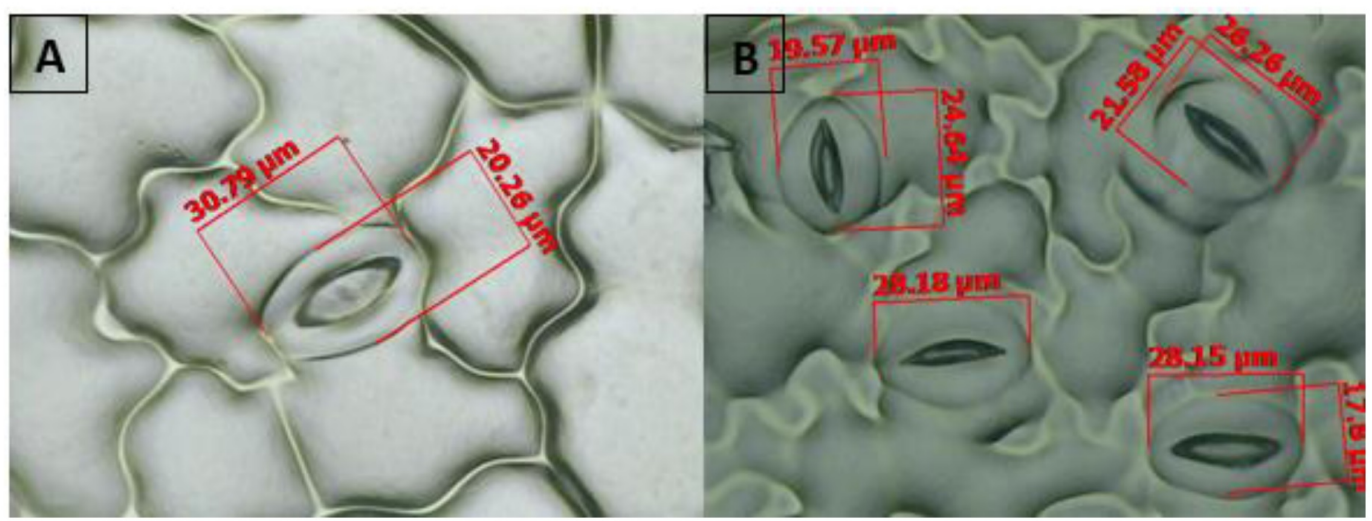

Figura 2. Impresiones de la epidermis de hojas de Solanum lycopersicum. Distribución y tamaño de estomas. (A) superficie adaxial y (B) superficie abaxial.

Tabla 2. Matriz de correlación entre las distintas variables evaluadas en las hojas 4 y 9.

\begin{tabular}{|c|c|c|c|c|c|c|c|c|c|c|c|c|c|c|c|}
\hline & & DEAb & DCAb & $\underset{\text { IEAb }}{\mathrm{H}}$ & $\begin{array}{c}O \\
\text { DEAd }\end{array}$ & $\begin{array}{c}\mathrm{J} \\
\text { DCAd }\end{array}$ & $\begin{array}{c}\text { A } \\
\text { IEAd }\end{array}$ & LEAb & $\begin{array}{c}9 \\
\text { AEAb }\end{array}$ & LEAd & AEAd & Tem & H.R. & DPV & RFA \\
\hline & DEAb & 1.00 & 0.26 & 0.84 & 0.16 & 0.47 & -0.05 & -0.54 & -0.26 & 0.15 & 0.16 & -0.16 & -0.38 & -0.27 & -0.67 \\
\hline & DCAb & 0.41 & 1.00 & -0.30 & 0.38 & 0.71 & 0.10 & -0.11 & -0.14 & 0.17 & -0.27 & 0.40 & 0.18 & -0.04 & 0.05 \\
\hline & IEAb & 0.88 & -0.05 & 1.00 & -0.04 & 0.08 & -0.09 & -0.47 & -0.14 & 0.08 & 0.31 & -0.37 & -0.46 & -0.22 & -0.68 \\
\hline & DEAd & -0.08 & 0.36 & -0.24 & 1.00 & 0.72 & 0.92 & 0.13 & 0.31 & 0.20 & 0.22 & 0.42 & -0.23 & -0.09 & 0.36 \\
\hline $\mathrm{H}$ & DCAd & 0.27 & 0.25 & 0.16 & 0.39 & 1.00 & 0.40 & -0.20 & -0.10 & 0.20 & 0.12 & 0.19 & -0.15 & -0.30 & -0.06 \\
\hline 0 & IEAd & -0.24 & 0.23 & -0.34 & 0.76 & -0.29 & 1.00 & 0.26 & 0.46 & 0.19 & 0.25 & 0.41 & -0.22 & 0.00 & 0.50 \\
\hline $\mathrm{J}$ & LEAb & -0.26 & -0.43 & -0.08 & -0.19 & -0.21 & -0.06 & 1.00 & 0.54 & -0.22 & -0.12 & 0.15 & 0.06 & 0.41 & 0.51 \\
\hline \multirow[t]{2}{*}{ A } & AEAb & -0.21 & -0.37 & -0.02 & 0.17 & -0.08 & 0.22 & 0.82 & 1.00 & -0.18 & 0.06 & 0.23 & 0.03 & 0.39 & 0.42 \\
\hline & LEAd & -0.43 & -0.38 & -0.24 & 0.10 & -0.20 & 0.22 & -0.01 & -0.03 & 1.00 & 0.59 & -0.25 & -0.15 & -0.36 & -0.15 \\
\hline \multirow[t]{5}{*}{4} & AEAd & -0.06 & 0.21 & -0.16 & 0.36 & -0.25 & 0.53 & 0.10 & 0.22 & -0.27 & 1.00 & -0.46 & -0.10 & -0.42 & -0.13 \\
\hline & Tem & -0.48 & -0.14 & -0.46 & 0.36 & -0.05 & 0.39 & -0.04 & -0.11 & 0.31 & 0.22 & 1.00 & 0.24 & 0.53 & 0.69 \\
\hline & H.R. & -0.25 & -0.09 & -0.32 & -0.16 & 0.16 & -0.26 & 0.05 & -0.14 & -0.19 & -0.01 & 0.24 & 1.00 & 0.24 & 0.28 \\
\hline & DPV & -0.34 & -0.21 & -0.26 & 0.00 & -0.01 & 0.04 & 0.24 & 0.06 & 0.31 & -0.27 & 0.53 & 0.24 & 1.00 & 0.47 \\
\hline & RFA & -0.78 & -0.42 & -0.64 & 0.10 & -0.20 & 0.24 & 0.09 & 0.01 & 0.62 & 0.09 & 0.69 & 0.28 & 0.47 & 1.00 \\
\hline
\end{tabular}

DEAb = densidad estomática abaxial; DEAd = densidad estomática adaxial; DCAb = densidad celular abaxial; DCAd = densidad celular adaxial; IEAb = índice estomático abaxial e IEAd = índice estomático adaxial; LEAb = largo de estomas abaxial; AEAb = ancho de estomas abaxial; LEAd = largo de estomas adaxial; $A E A d=$ ancho de estomas adaxial; $T e m=$ temperatura; H.R. = humedad relativa; $D P V=$ déficit de presión de vapor y RFA = radiación fotosintéticamente activa.

\section{LITERATURA CITADA}

Apaza MDA, Mestas VBR, Romero VFF, Navarro ORD (2019) Toxicidad del cobre sobre la morfología de estomas de Gochnatia arequipensis Sandwith (Asteraceae) de dos localidades de Arequipa, Perú. Idesia 37: 81-87.

Ayala AJ, Barrientos PAF, Colinas LMT, Sahagún CJ, Reyes AJC (2010) Relaciones injerto-interinjerto y características anatómicas y fisiológicas de la hoja de cuatro genotipos de aguacate. Revista Chapingo Serie Horticultura 16: 147-154.

Ayari O, Samson G, Dorais M, Boulanger R, Gosselin A (2000) Stomatal limitation of photosynthesis in winter production of greenhouse tomato plants. Physiologia Plantarum 110: 558-564.

Bazaldúa-Muñoz C, Ventura-Zapata E, Salcedo-Morales G, Maldonado AU, López GA (2008) Densidad estomatal y potencial hídrico en plantas de tomate (Physalis ixocarpa Brot.), propagadas por cultivo de meristemo. Revista Chapingo Serie Horticultura 14: 147-150.

Brownlee C (2001) The long and short of stomatal density signals. Trends in Plant Science 6: 441-442. 
Caballero AYS, de los Santos VS (2018) Origen de suelos depositados en la subcuenca del río Tapacalí, Madriz, Nicaragua mediante el uso de la técnica de isótopos estables de compuestos específicos. Aqua-LAC 10: 51-60.

Camposeco-Montejo N, Robledo-Torres V, Ramírez-Godina F, Valdez-Aguilar LA, Cabrera-de-la-Fuente M, Mendoza-Villareal R (2018) Efecto del portainjerto en el índice y densidad estomática de pimiento morrón Capsicum annuum var. annuum. Ecosistemas y Recursos Agropecuarios 5: 555-561.

Cañizares A, Sanabria ME, Rodríguez DA, Perozo Y (2003) Características de los estomas, índice y densidad estomática de las hojas de lima Tahití (Citrus latifolia Tanaka) injertada sobre ocho patrones cítricos. Revista Científica UDO Agrícola 3: 59-64.

De-las-Rivas J (2013) La luz y el aparato fotosintético. En: Azcon-Bieto J, Talón M (Eds.) Fundamentos de fisiología vegetal. McGraw-Hill. México. pp. 165-190.

Di Rienzo JA, Casanoves F, Balzarini MG, González L, Tablada M, Robledo, CW (2008) InfoStat versión 2008. Córdoba, Argentina: Grupo InfoStat, FCA, Universidad Nacional de Córdoba. https://www.infostat.com.ar/. Fecha de consulta: 01 de enero de 2021.

Esteban W, Pacheco P, Angel Y, Bastías E (2017) Efecto de la incorporación de materia orgánica en la respuesta fisiológica y química de plantas de tomate (Solanum lycopersicum L.) cultivadas en condiciones de salinidad y exceso de boro. Idesia 35: 33-39.

FIRA (2019) Panorama agroalimentario. Tomate rojo 2019. Dirección de Investigación y Evaluación Económica y Sectorial. Fideicomisos Instituidos en Relación con la Agricultura. México. 25p.

Flores-Vindas E (1999) La planta: estructura y función. Vol 1. Libro Universitario Regional. EULA/GTZ. Costa Rica. 884p.

Florido BM, Álvarez GM (2015) Aspectos relacionados con el estrés de calor en tomate (Solanum lycopersicum L.). Cultivos Tropicales 36: 77-95.

Gonzales P (2013) Geographical distribution of wild tomatos (Solanum L. sect. Lycopersicon (Mill.) Wettst.: Solanaceae). Arnaldoa 20: 301-314.

Hanssen I, Lapidot M, Thomma B (2010) Emerging viral diseases of tomato crops. The American Phytopathological Society 23: 539-548.

Hernández-Leal E, Lobato-Ortiz R, García-Zavala JJ, Reyes-López D, Méndez-López A, Bonilla-Barrientos O, Hernández-Bautista A (2013) Comportamiento agronómico de poblaciones F2 de híbridos de tomate (Solanum lycopersicum L.). Revista Fitotecnia Mexicana 36: 209-215.

INIA (2017) Manual de cultivo del tomate bajo invernadero. Instituto de Investigaciones Agropecuarias. Boletín INIA No12. 111p.

Misson L, Limousin JM, Rodriguez R, Letts MG (2010) Leaf physiological responses to extreme droughts in Mediterranean Quercus ilex forest. Plant, Cell \& Environment 33: 1898-1910.

Moya C, Oyanedel E, Verdugo G, Flores MF, Urrestarazu M, Álvaro JE (2017) Increased electrical conductivity in nutrient solution management enhances dietary and organoleptic qualities in soilless culture tomato. HortScience 52: 868-872.

Oyarzún R, Stöckle C, Wu J, Whiting M (2011) In field assessment on the relationship between photosynthetic active radiation (PAR) and global solar radiation transmittance through discontinuous canopies. Chilean Journal of Agricultural Research 71: 122-131. 
Petrova $Y$ (2012) The effect of light intensity on the stomatal density of lavender, Lavandula angustifolia. Young Scientists Journal 12: 89-93.

Rosenberg NJ, Blad BL, Verma SB (1983) Microclimate; the biological environment (2nd Edn). John Wiley and Sons. New York, USA. 528p.

Rueda-Barrientos MC, Martínez-Fernández E, Villegas-Torres OG, Sainz-Aispuro MJ, Peña-Chora G, HernándezVelazquez VM, Hernández-Romano J (2017) Sensibilidad de la prueba de InmunoStrips ${ }^{\circledR}$ en la detección de Clavibacter michiganensis subsp. michiganensis en tomate. Acta Agrícola y Pecuaria 3 (2): 50-57.

Salisbury FB, Ross CW (2000) Fisiología de las plantas. Thomson-Paraninfo. Madrid, España. 305p.

Sánchez-Díaz M, Aguirreolea J (2008) Transpiración y control estomático. En: Azcón-Bieto J, Talón M Fundamentos de fisiología vegetal. 2da Edición. McGraw-Hill Interamericana de España. Madrid, España. 669 .

Sato S, Peet MM (2005) Effects of moderately elevated temperature stress on the timing of pollen release and its germination in tomato (Lycopersicon esculentum Mill.). Journal of horticultural science \& Biotechnology 80 : 23-28.

Servicio Meteorológico Nacional (SMN) (2018) Normales climatológicas del estado de Coahuila de Zaragoza. Estación: 00005048 Saltillo (DGE). https://smn.conagua.gob.mx/tools/RESOURCES/Normales8110/NOR MAL05048.TXT. Fecha de consulta: 15 de enero de 2021.

SIAP (2014) Tomate rojo. Servicio de Información Agroalimentaria y Pesquera. http://www.siap.gob.mx/cierrede-la-produccion-agricola-por-cultivo. Fecha de consulta 6 de septiembre de 2020.

SIAP (2016) Jitomate (tomate rojo), es una hortaliza y sus propiedades son un medicamento natural. Servicio de Información Agroalimentaria y Pesquera. https://www.gob.mx/siap/articulos/jitomate-tomate-rojo-esuna-hortaliza-y-sus-propiedades-son-un-medicamento-natural. Fecha de consulta 19 de enero de 2021.

SIAP (2017) Atlas Agroalimentario 2017. Servicio de Información Agroalimentaria y Pesquera. http://nube.siap. gob.mx/gobmx_publicaciones_siap/pag/2017/Atlas-Agroalimentario-2017. Fecha de consulta 20 de agosto de 2021.

SIAP (2020) Servicio de Información Agroalimentaria y Pesquera. http://infosiap.siap.gob.mx:8080/agricola_siap _gobmx/AvanceNacionalCultivo.do. Fecha de consulta 19 de enero de 2021.

SIAVI (2020) Sistema de Información Arancelaria Vía Internet. http://www.economia-snci.gob.mx/. Fecha de consulta 19 julio de 2021.

Steiner AA (1961) A universal method for preparing nutrient solutions of a certain desired composition. Plant Soil 15: $134-154$.

Trewavas A (2003) Aspects of plant intelligence. Annals of Botany 92: 1-20.

Wang Z, Li G, Sun H, Ma L, Guo Y, Zhao Z, Gao H, Mei L (2018) Effects of drought stress on photosynthesis and photosynthetic electron transport chain in young apple tree leaves. Biol Open 7(11): bio035279. DOI: 10.1242/bio.035279.

Won-Sub L, Sung-Gaun K (2012) Development of Rotational Smart Lighting Control System for Plant Factory. International Scholarly and Scientific Research \& Innovation 6: 519-522. 